\title{
Comment on "The Surgical Management of Traumatic Lower Cervical Spondylolisthesis with Posterior Percutaneous Pedicle Screw Fixation”
}

\author{
Arjun RHH, Vijay Goni, Sarvadeep Dhatt, Vishal Kumar, Rakesh John, Uday Guled, Harsha Vardhan \\ Department of Orthopaedics, Post Graduate Institute of Medical Education and Research, Chandigarh, India
}

Dear Colleagues,

Your published technique evoked a great interest in us as it was discussed in one of the journal clubs at our institute. It was well received and appreciated; however, this innovative technique got few comments from the panel of experts. Below are the comments, which we thought could be worth a mention and might benefit the technique in the future: (1) The described technique mentioned that the K-wire was adjusted to the angle of 30 degrees in a sagittal plane to match the preoperatively measured pedicle angle, but there was no mention of any measuring tool intraoperatively to confirm the angle. We think this is an important step because the allowed margin of error is smaller in cervical pedicle screw fixation $(3.5 \mathrm{~mm})$ fixation due to narrow pedicles (5.9 to $6.7 \mathrm{~mm}$ of pedicle height and width at C7) [1] and proximity to vital structures (spinal cord, nerve roots, and vertebral artery) in the cervical region. Lack of pedicle angle confirmation intraoperatively might be a reason for misplaced pedicle screws in the described technique. We routinely use a goniometer for confirming the pedicle angle intraoperatively to match the preoperative measurement in our cervical pedicle screw insertion with an open technique.

(2) The statement " $5 \mathrm{~mm}$ incision was given and $7 \mathrm{~mm}$ protection sleeve inserted into it" in the technique description needs to be justified with clinical images.

(3) We suggest that percutaneous cervical pedicle insertion should not be tried without three-dimensional navigation technology.

\section{Conflict of Interest}

No potential conflict of interest relevant to this article was reported.

\section{Reference}

1. Mohi Eldin MM. Cervical pedicle screw fixation: anatomic feasibility of pedicle morphology and radiologic evaluation of the anatomical measurements. Asian Spine J 2014;8:273-80. 